\title{
Interplay of miRNAs and Canonical Wnt Signaling Pathway in Hepatocellular Carcinoma
}

\author{
Xiaobo Nie', Yiran Liu1,2, Wei-Dong Chen ${ }^{1,3 *}$ and Yan-Dong Wang ${ }^{4 *}$ \\ ${ }^{1}$ Key Laboratory of Receptors-Mediated Gene Regulation and Drug Discovery, School of Medicine, Henan University, \\ Kaifeng, China, ${ }^{2}$ Department of Pathology, Henan Provincial People's Hospital, Zhengzhou, China, ${ }^{3}$ Key Laboratory of \\ Molecular Pathology, School of Basic Medical Science, Inner Mongolia Medical University, Hohhot, China, ${ }^{4}$ State Key \\ Laboratory of Chemical Resource Engineering, College of Life Science and Technology, Beijing University of Chemical \\ Technology, Beijing, China
}

Hepatocellular carcinoma is one of the leading causes of cancer death worldwide and the activation of canonical Wnt signaling pathway is universal in hepatocellular carcinoma patients. MicroRNAs are found to participate in the pathogenesis of hepatocellular carcinoma by activating or inhibiting components in the canonical Wnt signaling pathway. Meanwhile, transcriptional activation of microRNAs by canonical Wnt signaling pathway also contributes to the occurrence and progression of hepatocellular carcinoma. Pharmacological inhibition of hepatocellular carcinoma pathogenesis and other cancers by microRNAs are now in clinical trials despite the challenges of identifying efficient microRNAs candidates and safe delivery vehicles. The focus of this review is on the interplay mechanisms between microRNAs and canonical Wnt signaling pathway in hepatocellular carcinoma, and a deep understanding of the crosstalk will promote to develop a better management of this disease.

Keywords: HCC, miRNA, Wnt, hepatocellular carcinoma, $\beta$-catenin

\section{INTRODUCTION}

Hepatocellular carcinoma (HCC) is the fifth most common malignancy and the second leading cause of cancer death worldwide, with more than 750,000 new cases diagnosed and 700,000 cancer deaths occurred annually. Approximately more than $80 \%$ of HCC-related deaths can be attributed to the chronic viral hepatitis infection (hepatitis $\mathrm{B}$ or $\mathrm{C}$ ), other risk factors including alcohol-related cirrhosis, non-alcoholic steatohepatitis, nitrosamine compounds or aflatoxin exposure, and algal toxins from contaminated water (Chuang et al., 1992; Ledda et al., 2017). The routine use of blood testing and image evaluation have obviously improved the detection of HCC, and improved living standard and childhood vaccination against hepatitis B have significantly reduced its incidence effectively in the past few decades (Blumberg et al., 1975). Unfortunately, the usual survival outcome of HCC is still poor, because it is often detected an advanced state or it is diagnosed after metastasis with no noticeable or unspecific symptom in the early stage. Hepatectomy and liver transplant are preferred therapies for HCC whereas they are limited to the liver function, and now a combination treatment including chemotherapy, radiation, interventional therapy and targeted therapy have been mostly applied and the survival rates of patients with HCC have been improved. However, there is no cure for HCC currently and it is still meaningful to explore detailed molecular mechanisms that contribute to improve diagnostic and therapeutic management of HCC. 
Hepatocellular carcinoma is a complex polygenetic disease ascribed to the interactions between genetic predisposition and environmental factors. Inactivation or loss of tumor suppressor genes such as p21, p53, Rb and PTEN and activation of oncogenes including AKT and N-ras could induce the carcinogenesis of HCC (Martin and Dufour, 2008; Ho et al., 2012). Meanwhile, HCC is also strongly correlated with abnormality of cellular signal pathways, including EGFR, VEGFR, MAPK, IGFR, Wnt signaling pathways (Whittaker et al., 2010; Berasain et al., 2011). Among them Wnt signaling pathways attract significant attention because its role in the pathogenesis of colorectal cancer has become clear (Bienz and Clevers, 2000), and more researches demonstrated that changes of Wnt signaling may be the common pathogenetic basis of cancers. Although it has been found that Wnt signaling pathway is mainly involved in the occurrence and development of HCC by activating its downstream target genes (Thompson and Monga, 2007), the function of components of Wnt signaling pathway within the HCC context remains partially understood.

MicroRNAs (miRNAs) are a class of short (18-25 nucleotides) non-coding RNAs that repress target gene expression through the interaction with the $3^{\prime}$-untranslated region $\left(3^{\prime}\right.$-UTR) of their target genes, leading to either mRNA degradation or the inhibition of mRNA translation. Studies have shown that miRNAs play important roles in the development of cancer including HCC (Zhang et al., 2007; Klingenberg et al., 2017), through acting on the corresponding target mRNAs involved in cellular proliferation, differentiation, apoptosis and metastasis. Previous studies have identified a number of miRNAs participating in the pathogenesis of HCC by regulating components in Wnt signaling pathway. In the recent days, Rahmani et al. (2017) reported the roles of $\mathrm{Wnt} / \beta$-catenin signaling pathway and related miRNAs in colorectal cancer. In this review, the crosstalk between miRNAs and Wnt signaling pathway in the pathogenesis of HCC is summarized.

\section{CANONICAL WNT SIGNALING PATHWAY IN HCC PATHOGENESIS}

Aberrant canonical Wnt signaling pathway is closely associated with the progression and metastasis of various cancers, including HCC, and gene mutations or abnormal expression of Wnt signaling components cause activation of canonical Wnt signaling pathway in HCC (Lee et al., 2006). For example, in a specific subtype of familial and sporadic hepatocellular carcinomas, DICER1 mutations in liver carcinogenesis are associated with CTNNB1 (encoding $\beta$-catenin) mutations and lead to the $\beta$-catenin activation (Caruso et al., 2017). Dickkopf 2 (DKK2) is an antagonist of Wnt3a, the reduction of its function due to the loss of DKK2 alleles leads to unchecked canonical Wnt signaling and contributes to HCC oncogenesis (Lin et al., 2016). In clinical HCC samples, CTNND1 ( $\delta$-catenin) expression was found to be up-regulated significantly in cancer tissues compared with paired normal liver tissues, and overexpression of CTNND1 in HCC cell lines promotes carcinous characters through indirectly enhancing $\mathrm{Wnt} / \beta$-catenin signaling
(Tang et al., 2016). Similarly, secreted frizzled-related protein-1 (SFRP1) is a well-known inhibitor of Wnt/ $\beta$-catenin signaling and patients with lower SFRP1 expression level in tumor tissue have poor overall survival rate in HCC (Davaadorj et al., 2016). In addition, $\mathrm{Wnt} / \beta$-catenin can be activated by epigenetic modifications, such as IncRNA or miRNA regulation, this review will focus on the interplay between canonical Wnt signaling and miRNAs in later sections, and we hope it will facilitate the development of improved therapies for HCC.

\section{MIRNAS TARGETING WNT LIGANDS/RECEPTORS AND ASSOCIATED INHIBITORY PROTEINS}

Wnt ligands are secreted as lipid-modified signaling glycoproteins comprising 19 family members in human being, and canonical Wnt signaling pathway is originally activated by the binding of Wnt ligands to its receptor such as FZD and LRP5 or LRP6. The pathway transduction will be interrupted if miRNAs target any of these Wnt ligands or receptors. miR122 expression level is found to be decreased significantly in human HCC tissue samples and cell lines, and overexpression of miR-122 inhibits proliferation but promotes hepatoma cell apoptosis by repressing Wnt1 expression, subsequently leads to blocking Wnt1/ $\beta$-catenin/TCF signaling pathway (Xu J. et al., 2012; Ahsani et al., 2017) (Table 1). Meanwhile, Wnt1 is also targeted by endogenous miR-148a in HCC cells. Yan et al. (2014) showed that miR-148a expression level in metastatic HCC tissues is lower than that of nonmetastatic ones, and overexpression of miR-148a blocks the metastasis of HCC cells by suppressing the epithelial-mesenchymal transition (EMT) and acquisition of cancer stem cells (CSCs)-like properties through affecting the canonical Wnt signaling pathway. Furthermore, miR-148b is confirmed as another miRNA regulating Wnt1. It is downregulated in human HCC tissues. Patients with higher miR-148b expression in tumor tissues are shown to have a better prognosis, therefore miR-148b functions as a tumor suppressor in HCC through targeting WNT1/ $\beta$-catenin pathway (Zhang J.G. et al., 2015) (Table 1). However, other Wnt family members have not been reported to be regulated directly by miRNAs in HCC.

Another group of miRNAs regulating Wnt receptors also act like tumor suppressors. miR-199a is frequently downregulated in HCC cells and tissues, and lower miR-199a expression in HCC tissues indicates malignant potential and poor prognosis. Restoration of miR-199a in HCC cells could inhibit cell proliferation and survival by targeting FZD7 and its downstream genes, including $\beta$-catenin, cyclin D1 and c-Myc (Song et al., 2014). Similarly, miR-27a expression is reported to be downregulated in the multidrug-resistant HCC cell line, and upregulation of miR-27a could enhance the sensitivity of HCC cells to chemotherapy drugs through targeting the FZD7/ $\beta$-catenin pathway (Chen Z. et al., 2013). Other miRNAs including miR-1269a, -202, -432, -126-3p and 610 could directly target LRP5 to inhibit the canonical Wnt signaling pathway. For example, miR-202 and miR-126-3p were proved to suppress cell proliferation, metastasis and angiogenesis of HCC by 
TABLE 1 | Oncogenic and tumor suppressor miRNAs targeting the components of canonical Wnt signaling pathways in the pathogenesis of HCC.

\begin{tabular}{|c|c|c|c|}
\hline miRNAs & $\begin{array}{l}\text { Targets in canonical } \\
\text { Wnt signaling }\end{array}$ & Effect & Reference \\
\hline \multicolumn{4}{|c|}{ Targeting Wnt ligands/receptors and associated proteins } \\
\hline miR-122 & Wnt1 & Tumor suppressor & Xu J. et al., 2012; Ahsani et al., 2017 \\
\hline miR-148a & Wnt1 & Tumor suppressor & Yan et al., 2014 \\
\hline miR-148b & Wnt1 & Tumor suppressor & Zhang J.G. et al., 2015 \\
\hline miR-152 & Wnt1 & Tumor suppressor & Huang et al., 2014 \\
\hline miR-27a & FZD7 & Tumor suppressor & Chen Z. et al., 2013 \\
\hline miR-199a & FZD7 & Tumor suppressor & Song et al., 2014 \\
\hline miR-1269a & LRP6 & Tumor suppressor & Min et al., 2017 \\
\hline miR-202 & LRP6 & Tumor suppressor & Zhang et al., 2014 \\
\hline miR-432 & LRP6 & Tumor suppressor & Jiang et al., 2015 \\
\hline $\operatorname{miR}-126-3 p$ & LRP6 & Tumor suppressor & Du et al., 2014 \\
\hline miR-610 & LRP6 & Tumor suppressor & Zeng et al., 2014 \\
\hline miR-181a & WIF-1 & Oncogene & Ji et al., 2014 \\
\hline miR-522 & DKK1, sFRP2 & Oncogene & Zhang H. et al., 2016 \\
\hline miR-200b & BMl1 & Tumor suppressor & Wu W.R. et al., 2016 \\
\hline miR-218 & BMl1 & Tumor suppressor & Fu et al., 2015 \\
\hline \multicolumn{4}{|c|}{ Targeting $\beta$-catenin } \\
\hline $\operatorname{miR}-214$ & $\beta$-catenin & Tumor suppressor & Wang et al., 2012; Xia et al., 2012 \\
\hline miR-200a & $\beta$-catenin & Tumor suppressor & Liu et al., 2013 \\
\hline miR-320a & $\beta$-catenin & Tumor suppressor & Lu et al., 2017 \\
\hline $\operatorname{miR}-338-3 p$ & $\beta$-catenin & Tumor suppressor & Zhang T. et al., 2016 \\
\hline miR-33a & $\beta$-catenin & Tumor suppressor & Fang et al., 2013 \\
\hline $\operatorname{miR}-153$ & WWOX ( $\beta$-catenin inhibitor) & Oncogene & Hua et al., 2015 \\
\hline miRNAs & Target in Wnt signaling & Effect & Reference \\
\hline \multicolumn{4}{|c|}{ Targeting $\beta$-catenin interacting proteins } \\
\hline miR-9 & E-cadherin (CDH1) & Oncogene & Tan et al., 2010; Drakaki et al., 2015 \\
\hline miR-106b & APC & Oncogene & Shen et al., 2013 \\
\hline $\operatorname{miR}-155$ & APC & Oncogene, & Zhang et al., 2012 \\
\hline miR-107 & AXIN2 & Oncogene & Zhang J.J. et al., 2015 \\
\hline $\mathrm{miR}-1246$ & AXIN2, GSK3 $\beta$ & Oncogene & Chai et al., 2016 \\
\hline miR-920 & $\beta$-TRCP & Tumor suppressor & Chen et al., 2010 \\
\hline \multicolumn{4}{|c|}{ Targeting transcription factors in canonical Wnt pathway } \\
\hline miR-139 & TCF-4 & Tumor suppressor & Gu et al., 2014 \\
\hline $\operatorname{miR}-30-5 p$ & BCL9 & tumor suppressor & Zhao et al., 2014 \\
\hline $\operatorname{miR}-101$ & NLK2 & Tumor suppressor & Shen et al., 2014 \\
\hline $\operatorname{miR}-181$ & NLK & Oncogene & Ji et al., 2009 \\
\hline $\operatorname{miR}-20 a-5 p$ & RUNX3 & Oncogene & Chen et al., 2016 \\
\hline miR-130a & RUNX3 & Oncogene & Xu N. et al., 2012 \\
\hline $\operatorname{miR}-129-2$ & SOX4 & Tumor suppressor & Chen X. et al., 2013 \\
\hline $\begin{array}{l}\text { miR-935, miR-24, } \\
\text { miR-184 }\end{array}$ & SOX7 & Oncogene & Ma et al., 2014; Wu et al., 2014; Liu et al., 2017 \\
\hline miR-221 & HDAC6 & Oncogene & Bae et al., 2015 \\
\hline \multicolumn{4}{|c|}{ targeting $\beta$-catenin responsive genes } \\
\hline miR-29b & MMP-2 & Tumor suppressor & Fang et al., 2011 \\
\hline $\operatorname{miR}-451$ & MMP-9 & Tumor suppressor & Arii et al., 1996 \\
\hline miR-199a & MMP-9 & Tumor suppressor & Zhang et al., 2013 \\
\hline $\operatorname{miR}-451$ & c-Myc & Tumor suppressor & Huang et al., 2015 \\
\hline miR-34a & c-Myc & Tumor suppressor & Xu et al., 2015 \\
\hline let-7a & c-Myc & Tumor suppressor & Deng et al., 2016 \\
\hline miR-199a-3p & CD44 & Tumor suppressor & Henry et al., 2010 \\
\hline miR-101 & $\mathrm{COX} 2$ & Tumor suppressor & Zheng et al., 2015 \\
\hline
\end{tabular}


TABLE 1 | Continued

\begin{tabular}{|c|c|c|c|}
\hline miRNAs & $\begin{array}{l}\text { Targets in canonical } \\
\text { Wnt signaling }\end{array}$ & Effect & Reference \\
\hline miR-19a & cyclin D1 & Tumor suppressor & Zhang Y. et al., 2015 \\
\hline miR-20a & cyclin D1 & Tumor suppressor & Karimkhanloo et al., 2017 \\
\hline miR-384 & IRS1- cyclin D1 & Tumor suppressor & Lai et al., 2016 \\
\hline
\end{tabular}

TABLE 2 | miRNAs regulated by canonical Wnt signaling pathways in the pathogenesis of HCC.

\begin{tabular}{|c|c|c|c|}
\hline miRNAs & Targeted by canonical & Effect of miRNAs & Reference \\
\hline \multicolumn{4}{|l|}{ Wnt signaling } \\
\hline miR-17-5p, miR-101 & c-Myc & Tumor suppressor & Wang et al., 2014; Liu et al., 2016 \\
\hline$m i R-148 a-5 p$, miR-363-3p & c-Myc & Tumor suppressor & Han et al., 2013 \\
\hline miR-181 & $\beta$-catenin/TCF4 & Oncogene & Ji et al., 2011 \\
\hline miR-183/96/182 & $\beta$-catenin/TCF3 & Oncogene & Leung et al., 2015 \\
\hline miR-770 & Wnt $/ \beta$-catenin & Oncogene & Wu W.J. et al., 2016 \\
\hline miR-122 & GSK-3 $\beta$ & Tumor suppressor & Zeng et al., 2010 \\
\hline miR-34a & $\beta$-catenin & Oncogene & Gougelet et al., 2016 \\
\hline
\end{tabular}

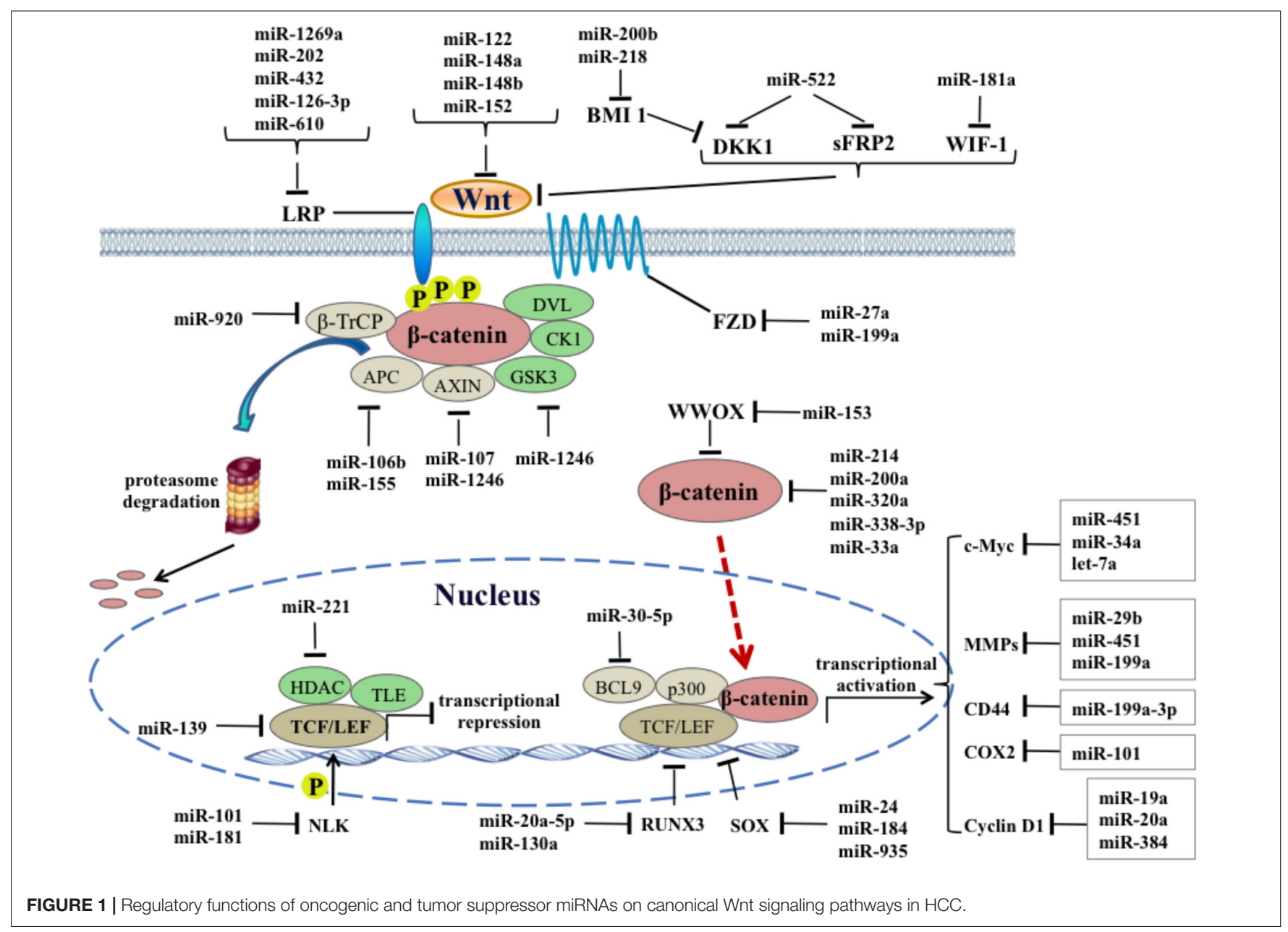

downregulating LRP6 expression (Du et al., 2014; Zhang et al., 2014). Inhibiting miR-610 and miR-432 enhances proliferation and tumorigenicity of HCC cells through directly suppressing multiple regulators of the $\mathrm{Wnt} / \mathrm{\beta}$-catenin signaling cascade, including LRP6 (Zeng et al., 2014; Jiang et al., 2015). Interestingly, a single nucleotide variant in miR-1269a could reduce its own 
anticancer effect and even promote the occurrence and process of HCC by disinhibiting the oncogene LRP6 (Min et al., 2017) (Figure 1).

Conversely, miRNAs targeting the inhibitory factors of Wnt ligands will be oncogenic and activates $\mathrm{Wnt} / \beta$-catenin pathway. As a secreted lipid binding protein that binds to Wnt proteins and inhibits Wnt signaling pathway, Wnt inhibitory factor1 (WIF-1) is identified as a direct and functional target of miR-181a in colorectal cancer, and an ectopic expression of miR-181a promotes tumor growth and liver metastasis (Ji et al., 2014). It can be speculated that WIF-1 could also be targeted by miR-181a due to the extensive low expression of WIF-1 and an opposite tendency of miR-181a in HCC (Deng et al., 2010). Dickkopf-1 (DKK1) and secreted frizzled-related protein 2 (SFRP2) are both antagonists of Wnt signaling. Sun's group has reported that miR-522 promotes cell proliferation of HCC by targeting DKK1 and SFRP2 and activating Wnt signaling (Zhang $\mathrm{H}$. et al., 2016), whereas other researchers report that DDK1 is overexpressed in HCC cells and tissues, and promotes HCC cell migration and invasion through $\beta$-catenin/MMP7 pathway (Chen L. et al., 2013). As an activator of the Wnt pathway by repressing DKK family, polycomb group protein BMI1 often overexpresses in HCC and is required for self-renewal of HCC CSC. In particular, BMI1 mediates activation of Wnt signaling, leading to a further transcriptional autoactivation of itself. Forced expression of miRNA-200b in HCC cells dramatically represses malignant features including cell proliferation, colony formation, and invasion by targeting BMI1 (Wu W.R. et al., 2016). Moreover, miR-218 is a tumor suppressor with an inhibitory effect on BMI1 expression in HCC pathology (Fu et al., 2015). Thus, any miRNAs that can regulate Wnt ligands/receptors and associate inhibitory proteins directly or indirectly could influence the transduction of the canonical Wnt signaling pathway.

\section{miRNAS TARGETING TRANSCRIPTION FACTORS IN CANONICAL WNT PATHWAY}

TCF/LEF transcription factors mediate a response to Wnt signals by binding to the stabilized $\beta$-catenin in nucleus, resulting in activation of Wnt-responsive genes. Negative regulation at the protein level of TCF/LEF transcription factors and associated coactivators/co-repressors by miRNAs will affect the signal transduction of $\mathrm{Wnt} / \beta$-catenin pathway and pathogenesis of HCC. TCF-4 promotes initiation and progression of cancers when binding to the $\beta$-catenin. However, in the absence of $\beta$-catenin, it will become a repressor of the transcription of $\beta$-catenin target genes by binding to co-repressors. In the previous study, a significant downregulation of $\mathrm{miR}$ 139 expression was observed in clinical HCC tissues, and miR-139 functions as an anticancer gene and suppresses the $\beta$-catenin/TCF-4 transcriptional activity by targeting TCF4 (Gu et al., 2014). BCL9 is a coactivator for TCF/LEF transcription factor and targeted by miR-30-5p, suppressing cancer progression in multiple myeloma cancers (Zhao et al., 2014), whereas its upstream miRNAs have not yet been found in HCC. As an evolutionarily conserved protein kinase involved in canonical Wnt signaling, nemo-like kinase (NLK) positively regulates $\mathrm{Wnt} / \beta$-catenin signalling by phosphorylating LEF1 and induces its dissociation from histone deacetylase, thereby allowing transcription activation (Ota et al., 2012). NLK2 is significantly overexpressed in HCC tissues and miR101 could inhibit multiple malignant phenotypes of HCC cells through regulating abnormal NLK2 activity (Shen et al., 2014). In contrast, NLK also appears to function as a tumor suppressor in HCC because a study has reported that miR181 induces HCC cell quantity and tumor initiating ability through direct inhibition of NLK mRNA translation (Ji et al., 2009).

\section{MIRNAS TARGETING $\beta$-CATENIN RESPONSIVE GENES IN CANONICAL WNT PATHWAY}

The regulation effect of canonical Wnt signaling pathway on tumorigenesis is achieved by changing the expression levels of diversified oncogenes that exhibit tissue-specific expression. So far, more than five hundred Wnt target genes have been identified by the gene chip and the whole genome ChIPsequencing, forming a rich Wnt target genome. MMPs, c-Myc, CD44, cyclin D1, and COX2, etc., are main target genes of canonical Wnt pathway in HCC and their roles have been intensively studied in the past decades. As the main enzymes that degrade extracellular matrix in the body, MMPs facilitate the invasion and metastasis of various malignant tumors including HCC. Overexpression of MMP-2 has been observed in patients with chronic hepatic disease and HCC tissues and a significant correlation exists between MMP-2 level and liver function (Kuyvenhoven et al., 2003). Zhuang et al. found that miR-29b suppresses tumor angiogenesis, venous invasion and metastasis, at least partially, by directly targeting MMP-2 expression in HCC (Fang et al., 2011). Similarly, the expression level of MMP-9 could be regarded as an indicator to detect the recurrence, invasion and metastasis of primary HCC, and overexpression of miR-451 abrogates human hepatoma cell growth and invasion, accompanied with the decrease of MMP-9 (Arii et al., 1996). Propofol, a commonly used intravenous anesthetics, was found to inhibit the invasiveness of HCC cells by elevating miR-199a expression and subsequent downregulating MMP-9 expression (Zhang et al., 2013) (Figure 1).

$\mathrm{c}-\mathrm{Myc}$ is a transcription factor that binds DNA in a nonspecific manner and regulates cell cycle positively. A recent study uncovered that the oncogene c-Myc is identified as a direct target of miR-451 and miR-451 downregulationinduced c-Myc overexpression leads to acquisition of EMT phenotype in HCC cells (Huang et al., 2015). In another HCC cell line, miR-34a induces cellular senescence via inhibiting the telomerase activity, at least partially by targeting $\mathrm{c}-\mathrm{Myc}$ (Xu et al., 2015). CD44 is described as a CSC marker in HCC 
and its expression is induced by canonical Wnt signaling, resulting in the recurrence and increased metastatic ability of HCC. miR-199a-3p was shown to reduce proliferation of CD44-positive HCC cell lines and sensitize the cells to chemotherapy drugs by repression of CD44 (Henry et al., 2010). COX-2 is highly expressed in well-differentiated HCC and can promote tumor cell growth and neovascularization through arresting cell cycle and blocking apoptosis. A recent study has shown that systemic delivery of lentivirus-mediated miR-101 strongly abrogated HCC tumorigenesis in the liver and intrahepatic and distant metastasis in vivo, by downregulating several targets including COX-2 (Zheng et al., 2015). Cycin D1 is a typical target gene in canonical Wnt pathway, its overexpression could accelerate the G1/S phase transition of cell cycle and cause uncontrolled cell proliferation. Recently, several studies revealed potential links between miRNAs and cyclin D1 in HCC. For example, a systematic investigation based on miRNA-mediated gene regulatory network revealed that miR-19a plays an inhibitory role in HCC via suppressing cyclin D1 expression, indicating therapeutics targeting miR-19a/cyclin D1 axis might be benefit for HCC (Zhang Y. et al., 2015). Moreover, bioinformatics prediction and experimental validation also proved that miR-20a could be another new therapeutic agent for HCC through targeting cyclin D1 (Karimkhanloo et al., 2017) (Figure 1).

\section{mIRNAs TARGETED BY CANONICAL WNT SIGNALING PATHWAY IN HCC}

Presently, more researches focus on the identification of novel miRNAs and their responsive targets in cancer, whereas the mechanisms by which miRNAs are activated or inhibited are obscure. It is interesting to find that components of canonical Wnt signaling pathway could conversely regulate miRNAs in HCC (Table 2). For instant, Wu et al. demonstrated that a regulatory feedback loop exists between miR-17-5p and c-Myc, in which miR-17-5p could inhibit metastasis and invasion of HCC cells by suppressing c-Myc, and miR-17$5 \mathrm{p}$, in turn, is induced by activated $\mathrm{c}-\mathrm{Myc}$ as a transcription factor, although detailed mechanism is still needed to be elucidated (Liu et al., 2016). Moreover, miR-101 is a direct target gene epigenetically silenced by c-Myc in HCC cells and overexpression of $\mathrm{c}-\mathrm{Myc}$ in HCC samples was closely related to lower miR-101 levels and poorer prognosis of HCC patients (Wang et al., 2014). Similarly, c-Myc is pathologically activated in HCC and induces hepatocarcinogenesis through a novel miRNA-mediated feedback loop comprised of miR148a-5p and miR-363-3p (Han et al., 2013). In addition to upregulating target genes in canonical Wnt signaling pathway, $\beta$-catenin/TCF4 complex could also activate the transcription of miRNAs and produce a positive feedback regulatory loop in HCC. Ji et al. (2011) reported that several putative $\beta$-catenin/TCF4 binding sites are identified in the promoter region of the miRNA-181a-2 and miRNA-181b-2 transcripts, and four members in miRNA-181 family are positively associated with $\beta$-catenin expression in HCC. This is in line with the previous report which demonstrates that miR-183/96/182 cluster is activated by $\mathrm{Wnt} / \beta$-catenin/TCF3 signaling in $\mathrm{HCC}$ and promotes cell migration and invasion (Leung et al., 2015). Besides, Wnt/ $\beta$-catenin signaling is found to act on the transcription of miRNA-770, subsequently exerting a positive influence on the tumorigenesis of HCC.

\section{PHARMACOLOGICAL INHIBITION OF HCC PATHOGENESIS BY MIRNAS}

The complicated and sophisticated regulation on abnormally expressed target genes during tumorigenesis makes miRNAs promising therapeutic agents or targets for cancer therapy. Functional studies indicated that downregulated tumor suppressor miRNAs could be restored to treat cancers by using miRNA mimics, and variety of antimiRs in the form of miRNA sponges, anti-miR oligonucleotides and small molecule inhibitors could be developed to inhibit or compete with oncogenic miRNA in preclinical development. Recently, several miRNA-targeted therapeutics have reached preclinical or clinical development for cancer therapy including HCC (Rupaimoole and Slack, 2017). MiR-155 is present at abnormally high levels in HCC and several other cancers, as a locked nucleic acid (LNA) to antagonize miR-155, MRG-106 is used for patients with certain lymphomas and leukemias and the ongoing Phase I clinical trial (ClinicalTrials.gov identifier: NCT02580552) which is available for assessing its safety, tolerability and pharmacokinetics will be completed in December 2018, thus the potential inhibition effect of MRG-106 on canonical Wnt signaling pathway will make it an excellent candidate for HCC therapy.

Chu et al. (2017) reported that miR-1247-5p has the potential to be a tumor suppressor in HCC by targeting Wnt3. It also has been reported that miR-337 regulates the $\mathrm{Wnt} / \beta$-catenin signaling pathway to inhibit HCC progression by targeting high-mobility group AT-hook 2 (Cui et al., 2018). miR221 is one of the most significantly upregulated miRNAs in solid tumors such as HCC, pancreas and lung cancers. It targets the tumor suppressors such as PTEN, metalloproteinase inhibitor 3 (TIMP3) and HDAC6 in canonical Wnt signaling (Garofalo et al., 2009; Bae et al., 2015). Intravenously delivering to a valid orthotopic mouse model of HCC blocks tumor cell proliferation and elevates mouse survival. Furthermore, a cholesterol-modified form of anti-miR-221 exhibits improved liver tissue distribution and pharmacokinetics than unmodified oligonucleotide, suggesting a broad application prospects for patients with HCC (Park et al., 2011). These reports support the potentiality for miRNAs as targets for treatment of HCC in the future.

At present, several main challenges are still blocking the movement of miRNAs therapeutics into clinically development, including the identification of the best miRNAs or targets for specific cancer type, off-target effect due to the similar sequence of miRNAs within the same family, severe toxicity 
and immunogenicity of chemically modified miRNAs, low bioavailability and delivery efficiency of miRNA delivery vehicles. Additionally, one miRNA can regulate serial different genes and its inhibition can affect undesirably other biological pathways. However, with the advances in biotechnology and great efforts of scientists, miRNA-based therapeutics are still promising to become the clinical reality for cancer treatment.

\section{CONCLUSION}

It is well known that oncogenic or tumor suppressor miRNAs modulates the HCC cell proliferation, invasion, metastasis and drug susceptibility, through targeting components or regulatory factors in canonical Wnt signaling pathway. Conversely, feedback regulation of miRNAs by canonical Wnt signaling pathway could also contribute to the pathogenesis of HCC. In this review, we summarize the recent findings on the interaction between miRNAs and canonical Wnt signaling pathway in HCC, and it remains to be deeply investigated the specific upstream regulators for the miRNAs that target this pathway. But all in all, miRNAbased therapeutics for HCC and other cancers are now in clinical trials despite plenty of technical challenges, and increased knowledge about the interplay between miRNAs and canonical Wnt signaling pathway will reveal the underlying mechanisms of HCC and contribute to novel miRNAs-based therapeutic designs.

\section{REFERENCES}

Ahsani, Z., Mohammadi-Yeganeh, S., Kia, V., Karimkhanloo, H., Zarghami, N., and Paryan, M. (2017). WNT1 gene from WNT signaling pathway is a direct target of miR-122 in hepatocellular carcinoma. Appl. Biochem. Biotechnol. 181, 884-897. doi: 10.1007/s12010-016-2256-8

Arii, S., Mise, M., Harada, T., Furutani, M., Ishigami, S., Niwano, M., et al. (1996). Overexpression of matrix metalloproteinase 9 gene in hepatocellular carcinoma with invasive potential. Hepatology 24, 316-322. doi: 10.1002/hep.510240206

Bae, H. J., Jung, K. H., Eun, J. W., Shen, Q., Kim, H. S., Park, S. J., et al. (2015). MicroRNA-221 governs tumor suppressor HDAC6 to potentiate malignant progression of liver cancer. J. Hepatol. 63, 408-419. doi: 10.1016/j.jhep.2015. 03.019

Berasain, C., Ujue Latasa, M., Urtasun, R., Goni, S., Elizalde, M., GarciaIrigoyen, O., et al. (2011). Epidermal growth factor receptor (EGFR) crosstalks in liver cancer. Cancers 3, 2444-2461. doi: 10.3390/cancers3022444

Bienz, M., and Clevers, H. (2000). Linking colorectal cancer to Wnt signaling. Cell 103, 311-320. doi: 10.1016/S0092-8674(00)00122-7

Blumberg, B. S., Larouze, B., London, W. T., Werner, B., Hesser, J. E., Millman, I., et al. (1975). The relation of infection with the hepatitis B agent to primary hepatic carcinoma. Am. J. Pathol. 81, 669-682.

Caruso, S., Calderaro, J., Letouze, E., Nault, J. C., Couchy, G., Boulai, A., et al. (2017). Germline and somatic DICER1 mutations in familial and sporadic liver tumors. J. Hepatol. 66, 734-742. doi: 10.1016/j.jhep.2016.12.010

Chai, S., Ng, K. Y., Tong, M., Lau, E. Y., Lee, T. K., Chan, K. W., et al. (2016). Octamer 4/microRNA-1246 signaling axis drives Wnt/beta-catenin activation in liver cancer stem cells. Hepatology 64, 2062-2076. doi: 10.1002/hep.28821

Chen, L., Li, M., Li, Q., Wang, C. J., and Xie, S. Q. (2013). DKK1 promotes hepatocellular carcinoma cell migration and invasion through betacatenin/MMP7 signaling pathway. Mol. Cancer 12:157. doi: 10.1186/14764598-12-157

Chen, S., He, Y., Ding, J., Jiang, Y., Jia, S., Xia, W., et al. (2010). An insertion/deletion polymorphism in the 3 ' untranslated region of

\section{AUTHOR CONTRIBUTIONS}

$\mathrm{XN}$ wrote the manuscript. YL edited the manuscript. W-DC and Y-DW edited and modified the manuscript.

\section{FUNDING}

This work was supported by the National Natural Science Foundation of China (Grant Nos. 81472232 and 81270522) to W-DC, and Henan Provincial Natural Science Foundation (Grant No. 162300410034), the National Natural Science Foundation of China (Grant No. 81700731), the Scientific Research Fund of Henan University (Grant No. 2015YBZR051) to XN, the National Natural Science Foundation of China (Grant Nos. 81672433 and 81370537) to Y-DW, Program for Science \& Technology Innovation Talents in Universities of Henan Province (HASTIT, Grant No. 13HASTIT024) and Plan for Scientific Innovation Talent of Henan Province to W-DC, and the Fundamental Research Funds for the Central Universities (Grant Nos. PT1624 and PYBZ1706) to Y-DW.

\section{ACKNOWLEDGMENTS}

We apologize to colleagues whose work could not be cited due to space limitations.

beta-transducin repeat-containing protein (betaTrCP) is associated with susceptibility for hepatocellular carcinoma in Chinese. Biochem. Biophys. Res. Commun. 391, 552-556. doi: 10.1016/j.bbrc.2009.11.096

Chen, X., Zhang, L., Zhang, T., Hao, M., Zhang, X., Zhang, J., et al. (2013). Methylation-mediated repression of microRNA 129-2 enhances oncogenic SOX4 expression in HCC. Liver Int. 33, 476-486. doi: 10.1111/liv. 12097

Chen, Y., Wang, X., Cheng, J., Wang, Z., Jiang, T., Hou, N., et al. (2016). MicroRNA-20a-5p targets RUNX3 to regulate proliferation and migration of human hepatocellular cancer cells. Oncol. Rep. 36, 3379-3386. doi: 10.3892/or. 2016.5144

Chen, Z., Ma, T., Huang, C., Zhang, L., Lv, X., Xu, T., et al. (2013). MiR-27a modulates the MDR1/P-glycoprotein expression by inhibiting FZD7/betacatenin pathway in hepatocellular carcinoma cells. Cell Signal. 25, 2693-2701. doi: 10.1016/j.cellsig.2013.08.032

Chu, Y., Fan, W., Guo, W., Zhang, Y., Wang, L., Guo, L., et al. (2017). miR-1247$5 \mathrm{p}$ functions as a tumor suppressor in human hepatocellular carcinoma by targeting Wnt3. Oncol. Rep. 38, 343-351. doi: 10.3892/or.2017.5702

Chuang, W. L., Chang, W. Y., Lu, S. N., Su, W. P., Lin, Z. Y., Chen, S. C., et al. (1992). The role of hepatitis B and C viruses in hepatocellular carcinoma in a hepatitis B endemic area. A case-control study. Cancer 69, 2052-2054. doi: $\quad$ 10.1002/1097-0142(19920415)69:8<2052::AID-CNCR2820690808>3.0. $\mathrm{CO} ; 2-\mathrm{N}$

Cui, H., Song, R., Wu, J., Wang, W., Chen, X., and Yin, J. (2018). MicroRNA337 regulates the PI3K/AKT and Wnt/beta-catenin signaling pathways to inhibit hepatocellular carcinoma progression by targeting high-mobility group AT-hook 2. Am. J. Cancer Res. 8, 405-421.

Davaadorj, M., Imura, S., Saito, Y. U., Morine, Y., Ikemoto, T., Yamada, S., et al. (2016). Loss of SFRP1 expression is associated with poor prognosis in hepatocellular carcinoma. Anticancer Res. 36, 659-664.

Deng, M., Hou, J., Hu, J., Wang, S., Chen, M., Chen, L., et al. (2016). virus mRNAs functionally sequester let-7a and enhance hepatocellular carcinoma. Cancer Lett. 383, 62-72. doi: 10.1016/j.canlet.2016.09.028 
Deng, Y., Yu, B., Cheng, Q., Jin, J., You, H., Ke, R., et al. (2010). Epigenetic silencing of WIF-1 in hepatocellular carcinomas. J. Cancer Res. Clin. Oncol. 136, 1161-1167. doi: 10.1007/s00432-010-0763-5

Drakaki, A., Hatziapostolou, M., Polytarchou, C., Vorvis, C., Poultsides, G. A., Souglakos, J., et al. (2015). Functional microRNA high throughput screening reveals miR-9 as a central regulator of liver oncogenesis by affecting the PPARA-CDH1 pathway. BMC Cancer 15:542. doi: 10.1186/s12885-015-1562-9

Du, C., Lv, Z., Cao, L., Ding, C., Gyabaah, O. A., Xie, H., et al. (2014). MiR-126-3p suppresses tumor metastasis and angiogenesis of hepatocellular carcinoma by targeting LRP6 and PIK3R2. J. Transl. Med. 12:259. doi: 10.1186/s12967-0140259- 1

Fang, J. H., Zhou, H. C., Zeng, C., Yang, J., Liu, Y., Huang, X., et al. (2011). MicroRNA-29b suppresses tumor angiogenesis, invasion, and metastasis by regulating matrix metalloproteinase 2 expression. Hepatology 54, 1729-1740. doi: 10.1002/hep.24577

Fang, Y., Feng, Y., Wu, T., Srinivas, S., Yang, W., Fan, J., et al. (2013). Aflatoxin $\mathrm{B} 1$ negatively regulates $\mathrm{Wnt} /$ beta-catenin signaling pathway through activating miR-33a. PLoS One 8:e73004. doi: 10.1371/journal.pone.0073004

Fu, Z. D., Cui, J. Y., and Klaassen, C. D. (2015). The role of sirt1 in bile acid regulation during calorie restriction in mice. PLoS One 10:e138307. doi: 10. 1371/journal.pone.0138307

Garofalo, M., Di Leva, G., Romano, G., Nuovo, G., Suh, S. S., Ngankeu, A., et al. (2009). miR-221\&222 regulate TRAIL resistance and enhance tumorigenicity through PTEN and TIMP3 downregulation. Cancer Cell 16, 498-509. doi: 10. 1016/j.ccr.2009.10.014

Gougelet, A., Sartor, C., Bachelot, L., Godard, C., Marchiol, C., Renault, G., et al. (2016). Antitumour activity of an inhibitor of miR-34a in liver cancer with beta-catenin-mutations. Gut 65, 1024-1034. doi: 10.1136/gutjnl-2014308969

Gu, W., Li, X., and Wang, J. (2014). miR-139 regulates the proliferation and invasion of hepatocellular carcinoma through the WNT/TCF-4 pathway. Oncol. Rep. 31, 397-404. doi: 10.3892/or.2013.2831

Han, H., Sun, D., Li, W., Shen, H., Zhu, Y., Li, C., et al. (2013). A c-MycMicroRNA functional feedback loop affects hepatocarcinogenesis. Hepatology 57, 2378-2389. doi: 10.1002/hep.26302

Henry, J. C., Park, J. K., Jiang, J., Kim, J. H., Nagorney, D. M., Roberts, L. R., et al. (2010). miR-199a-3p targets CD44 and reduces proliferation of CD44 positive hepatocellular carcinoma cell lines. Biochem. Biophys. Res. Commun. 403, 120-125. doi: 10.1016/j.bbrc.2010.10.130

Ho, C., Wang, C., Mattu, S., Destefanis, G., Ladu, S., Delogu, S., et al. (2012). AKT (v-akt murine thymoma viral oncogene homolog 1) and N-Ras (neuroblastoma ras viral oncogene homolog) coactivation in the mouse liver promotes rapid carcinogenesis by way of mTOR (mammalian target of rapamycin complex 1), FOXM1 (forkhead box M1)/SKP2, and c-Myc pathways. Hepatology 55, 833-845. doi: 10.1002/hep.24736

Hua, H. W., Jiang, F., Huang, Q., Liao, Z., and Ding, G. (2015). MicroRNA-153 promotes Wnt/beta-catenin activation in hepatocellular carcinoma through suppression of WWOX. Oncotarget 6, 3840-3847. doi: 10.18632/oncotarget. 2927

Huang, J. Y., Zhang, K., Chen, D. Q., Chen, J., Feng, B., Song, H., et al. (2015). MicroRNA-451: epithelial-mesenchymal transition inhibitor and prognostic biomarker of hepatocelluar carcinoma. Oncotarget 6, 18613-18630. doi: 10. 18632/oncotarget.4317

Huang, S., Xie, Y., Yang, P., Chen, P., and Zhang, L. (2014). HCV core proteininduced down-regulation of microRNA-152 promoted aberrant proliferation by regulating Wnt1 in HepG2 cells. PLoS One 9:e81730. doi: 10.1371/journal. pone. 0081730

Ji, D., Chen, Z., Li, M., Zhan, T., Yao, Y., Zhang, Z., et al. (2014). MicroRNA-181a promotes tumor growth and liver metastasis in colorectal cancer by targeting the tumor suppressor WIF-1. Mol. Cancer 13, 86. doi: 10.1186/1476-459813-86

Ji, J., Yamashita, T., Budhu, A., Forgues, M., Jia, H. L., Li, C., et al. (2009). Identification of microRNA-181 by genome-wide screening as a critical player in EpCAM-positive hepatic cancer stem cells. Hepatology 50, 472-480. doi: 10.1002/hep.22989

Ji, J., Yamashita, T., and Wang, X. W. (2011). Wnt/beta-catenin signaling activates microRNA-181 expression in hepatocellular carcinoma. Cell Biosci. 1:4. doi: 10.1186/2045-3701-1-4
Jiang, N., Chen, W. J., Zhang, J. W., Xu, C., Zeng, X. C., Zhang, T., et al. (2015). Downregulation of miR-432 activates Wnt/beta-catenin signaling and promotes human hepatocellular carcinoma proliferation. Oncotarget 6, 7866-7879.

Karimkhanloo, H., Mohammadi-Yeganeh, S., Ahsani, Z., and Paryan, M. (2017). Bioinformatics prediction and experimental validation of microRNA20a targeting Cyclin D1 in hepatocellular carcinoma. Tumour Biol. 39, 1010428317698361. doi: 10.1177/1010428317698361

Klingenberg, M., Matsuda, A., Diederichs, S., and Patel, T. (2017). Non-coding RNA in hepatocellular carcinoma: Mechanisms, biomarkers and therapeutic targets. J. Hepatol. 67, 603-618. doi: 10.1016/j.jhep.2017.04.009

Kuyvenhoven, J. P., van Hoek, B., Blom, E., van Duijn, W., Hanemaaijer, R., Verheijen, J. H., et al. (2003). Assessment of the clinical significance of serum matrix metalloproteinases MMP-2 and MMP-9 in patients with various chronic liver diseases and hepatocellular carcinoma. Thromb. Haemost. 89, 718-725. doi: 10.1055/s-0037-1613578

Lai, Y. Y., Shen, F., Cai, W. S., Chen, J. W., Feng, J. H., Cao, J., et al. (2016). MiR-384 regulated IRS1 expression and suppressed cell proliferation of human hepatocellular carcinoma. Tumour Biol. 37, 14165-14171. doi: 10.1007/s13277016-5233-5

Ledda, C., Loreto, C., Zammit, C., Marconi, A., Fago, L., Matera, S., et al. (2017). Noninfective occupational risk factors for hepatocellular carcinoma: a review (Review). Mol. Med. Rep. 15, 511-533. doi: 10.3892/mmr.2016.6046

Lee, H. C., Kim, M., and Wands, J. R. (2006). Wnt/Frizzled signaling in hepatocellular carcinoma. Front. Biosci. 11:1901-1915. doi: 10.2741/1933

Leung, W. K., He, M., Chan, A. W., Law, P. T., and Wong, N. (2015). Wnt/betaCatenin activates MiR-183/96/182 expression in hepatocellular carcinoma that promotes cell invasion. Cancer Lett 362, 97-105. doi: 10.1016/j.canlet.2015.03. 023

Lin, Y. F., Li, L. H., Lin, C. H., Tsou, M. H., Chuang, M. T., Wu, K. M., et al. (2016). Selective retention of an inactive allele of the DKK2 tumor suppressor gene in hepatocellular carcinoma. PLoS Genet. 12:e1006051. doi: 10.1371/journal.pgen. 1006051

Liu, D., Dong, L., Liu, Y., Wen, D., Gao, D., Sun, H., et al. (2016). A c-Myc/miR-17$5 \mathrm{p}$ feedback loop regulates metastasis and invasion of hepatocellular carcinoma. Tumour Biol. 37, 5039-5047. doi: 10.1007/s13277-015-4355-5

Liu, J., Ruan, B., You, N., Huang, Q., Liu, W., Dang, Z., et al. (2013). Downregulation of miR-200a induces EMT phenotypes and CSC-like signatures through targeting the beta-catenin pathway in hepatic oval cells. PLoS One 8:e79409. doi: 10.1371/journal.pone.0079409

Liu, X., Li, J., Yu, Z., Li, J., Sun, R., and Kan, Q. (2017). miR-935 promotes liver cancer cell proliferation and migration by targeting SOX7. Oncol. Res. 25, 427-435. doi: 10.3727/096504016X14747300207374

Lu, C., Liao, Z., Cai, M., and Zhang, G. (2017). MicroRNA-320a downregulation mediates human liver cancer cell proliferation through the Wnt/beta-catenin signaling pathway. Oncol. Lett. 13, 573-578. doi: 10.3892/ol.2016.5479

Ma, Y., She, X. G., Ming, Y. Z., and Wan, Q. Q. (2014). miR-24 promotes the proliferation and invasion of HCC cells by targeting SOX7. Tumour Biol. 35, 10731-10736. doi: 10.1007/s13277-014-2018-6

Martin, J., and Dufour, J. F. (2008). Tumor suppressor and hepatocellular carcinoma. World J. Gastroenterol. 14, 1720-1733. doi: 10.3748/wjg.14.1720

Min, P., Li, W., Zeng, D., Ma, Y., Xu, D., Zheng, W., et al. (2017). A single nucleotide variant in microRNA-1269a promotes the occurrence and process of hepatocellular carcinoma by targeting to oncogenes SPATS2L and LRP6. Bull. Cancer 104, 311-320. doi: 10.1016/j.bulcan.2016.11.021

Ota, S., Ishitani, S., Shimizu, N., Matsumoto, K., Itoh, M., and Ishitani, T. (2012). NLK positively regulates Wnt/beta-catenin signalling by phosphorylating LEF1 in neural progenitor cells. EMBO J. 31, 1904-1915. doi: 10.1038/emboj.2012.46

Park, J. K., Kogure, T., Nuovo, G. J., Jiang, J., He, L., Kim, J. H., et al. (2011). miR221 silencing blocks hepatocellular carcinoma and promotes survival. Cancer Res. 71, 7608-7616. doi: 10.1158/0008-5472.CAN-11-1144

Rahmani, F., Avan, A., Hashemy, S. I., and Hassanian, S. M. (2017). Role of Wnt/beta-catenin signaling regulatory microRNAs in the pathogenesis of colorectal cancer. J. Cell. Physiol. 233, 811-817. doi: 10.1002/jcp. 25897

Rupaimoole, R., and Slack, F. J. (2017). MicroRNA therapeutics: towards a new era for the management of cancer and other diseases. Nat. Rev. Drug Discov. 16, 203-222. doi: 10.1038/nrd.2016.246 
Shen, G., Jia, H., Tai, Q., Li, Y., and Chen, D. (2013). miR-106b downregulates adenomatous polyposis coli and promotes cell proliferation in human hepatocellular carcinoma. Carcinogenesis 34, 211-219. doi: 10.1093/carcin/ bgs320

Shen, Q., Bae, H. J., Eun, J. W., Kim, H. S., Park, S. J., Shin, W. C., et al. (2014). MiR101 functions as a tumor suppressor by directly targeting nemo-like kinase in liver cancer. Cancer Lett. 344, 204-211. doi: 10.1016/j.canlet.2013.10.030

Song, J., Gao, L., Yang, G., Tang, S., Xie, H., Wang, Y., et al. (2014). MiR-199a regulates cell proliferation and survival by targeting FZD7. PLoS One 9:e110074. doi: 10.1371 /journal.pone.0110074

Tan, H. X., Wang, Q., Chen, L. Z., Huang, X. H., Chen, J. S., Fu, X. H., et al. (2010). MicroRNA-9 reduces cell invasion and E-cadherin secretion in SK-Hep-1 cell. Med. Oncol. 27, 654-660. doi: 10.1007/s12032-009-9264-2

Tang, B., Tang, F., Wang, Z., Qi, G., Liang, X., Li, B., et al. (2016). Overexpression of CTNND1 in hepatocellular carcinoma promotes carcinous characters through activation of Wnt/beta-catenin signaling. J. Exp. Clin. Cancer Res. 35:82. doi: 10.1186/s13046-016-0344-9

Thompson, M. D., and Monga, S. P. (2007). WNT/beta-catenin signaling in liver health and disease. Hepatology 45, 1298-1305. doi: 10.1002/hep.21651

Wang, L., Zhang, X., Jia, L. T., Hu, S. J., Zhao, J., Yang, J. D., et al. (2014). c-Mycmediated epigenetic silencing of MicroRNA-101 contributes to dysregulation of multiple pathways in hepatocellular carcinoma. Hepatology 59, 1850-1863. doi: $10.1002 /$ hep. 26720

Wang, X., Chen, J., Li, F., Lin, Y., Zhang, X., Lv, Z., et al. (2012). MiR-214 inhibits cell growth in hepatocellular carcinoma through suppression of beta-catenin. Biochem. Biophys. Res. Commun. 428, 525-531. doi: 10.1016/j.bbrc.2012.10.039

Whittaker, S., Marais, R., and Zhu, A. X. (2010). The role of signaling pathways in the development and treatment of hepatocellular carcinoma. Oncogene 29, 4989-5005. doi: 10.1038/onc.2010.236

Wu, G. G., Li, W. H., He, W. G., Jiang, N., Zhang, G. X., Chen, W., et al. (2014). Mir-184 post-transcriptionally regulates SOX7 expression and promotes cell proliferation in human hepatocellular carcinoma. PLoS One 9:e88796. doi: 10. 1371/journal.pone.0088796

Wu, W. J., Shi, J., Hu, G., Yu, X., Lu, H., Yang, M. L., et al. (2016). Wnt/beta-catenin signaling inhibits FBXW7 expression by upregulation of microRNA-770 in hepatocellular carcinoma. Tumour Biol. 37, 6045-6051. doi: 10.1007/s13277015-4452-5

Wu, W. R., Sun, H., Zhang, R., Yu, X. H., Shi, X. D., Zhu, M. S., et al. (2016). Methylation-associated silencing of miR-200b facilitates human hepatocellular carcinoma progression by directly targeting BMI1. Oncotarget 7, 18684-18693. doi: 10.18632/oncotarget.7629

Xia, H., Ooi, L. L., and Hui, K. M. (2012). MiR-214 targets beta-catenin pathway to suppress invasion, stem-like traits and recurrence of human hepatocellular carcinoma. PLoS One 7:e44206. doi: 10.1371/journal.pone.0044206

Xu, J., Zhu, X., Wu, L., Yang, R., Yang, Z., Wang, Q., et al. (2012). MicroRNA122 suppresses cell proliferation and induces cell apoptosis in hepatocellular carcinoma by directly targeting Wnt/beta-catenin pathway. Liver Int. 32, 752-760. doi: 10.1111/j.1478-3231.2011.02750.x

Xu, N., Shen, C., Luo, Y., Xia, L., Xue, F., Xia, Q., et al. (2012). Upregulated miR-130a increases drug resistance by regulating RUNX3 and Wnt signaling in cisplatin-treated HCC cell. Biochem. Biophys. Res. Commun. 425, 468-472. doi: 10.1016/j.bbrc.2012.07.127

Xu, X., Chen, W., Miao, R., Zhou, Y., Wang, Z., Zhang, L., et al. (2015). miR-34a induces cellular senescence via modulation of telomerase activity in human hepatocellular carcinoma by targeting FoxM1/c-Myc pathway. Oncotarget 6, 3988-4004. doi: 10.18632/oncotarget.2905

Yan, H., Dong, X., Zhong, X., Ye, J., Zhou, Y., Yang, X., et al. (2014). Inhibitions of epithelial to mesenchymal transition and cancer stem cells-like properties are involved in miR-148a-mediated anti-metastasis of hepatocellular carcinoma. Mol. Carcinog. 53, 960-969. doi: 10.1002/mc.22064
Zeng, C., Wang, R., Li, D., Lin, X. J., Wei, Q. K., Yuan, Y., et al. (2010). A novel GSK-3 beta-C/EBP alpha-miR-122-insulin-like growth factor 1 receptor regulatory circuitry in human hepatocellular carcinoma. Hepatology 52, 1702-1712. doi: 10.1002/hep.23875

Zeng, X. C., Liu, F. Q., Yan, R., Yi, H. M., Zhang, T., Wang, G. Y., et al. (2014). Downregulation of miR-610 promotes proliferation and tumorigenicity and activates Wnt/beta-catenin signaling in human hepatocellular carcinoma. Mol. Cancer 13:261. doi: 10.1186/1476-4598-13-261

Zhang, B., Pan, X., Cobb, G. P., and Anderson, T. A. (2007). microRNAs as oncogenes and tumor suppressors. Dev. Biol. 302, 1-12. doi: 10.1016/j.ydbio. 2006.08.028

Zhang, H., Yu, C., Chen, M., Li, Z., Tian, S., Jiang, J., et al. (2016). miR-522 contributes to cell proliferation of hepatocellular carcinoma by targeting DKK1 and SFRP2. Tumour Biol. 37, 11321-11329. doi: 10.1007/s13277-016-4995-0

Zhang, J., Zhang, D., Wu, G. Q., Feng, Z. Y., and Zhu, S. M. (2013). Propofol inhibits the adhesion of hepatocellular carcinoma cells by upregulating microRNA-199a and downregulating MMP-9 expression. Hepatobiliary Pancreat. Dis. Int. 12, 305-309. doi: 10.1016/S1499-3872(13)60048-X

Zhang, J. G., Shi, Y., Hong, D. F., Song, M., Huang, D., Wang, C. Y., et al. (2015). MiR-148b suppresses cell proliferation and invasion in hepatocellular carcinoma by targeting WNT1/beta-catenin pathway. Sci. Rep. 5:8087. doi: $10.1038 /$ srep08087

Zhang, J. J., Wang, C. Y., Hua, L., Yao, K. H., Chen, J. T., and Hu, J. H. (2015). miR107 promotes hepatocellular carcinoma cell proliferation by targeting Axin2. Int. J. Clin. Exp. Pathol. 8, 5168-5174.

Zhang, T., Liu, W., Zeng, X. C., Jiang, N., Fu, B. S., Guo, Y., et al. (2016). Downregulation of microRNA-338-3p promoted angiogenesis in hepatocellular carcinoma. Biomed. Pharmacother. 84, 583-591. doi: 10.1016/j.biopha.2016.09. 056

Zhang, Y., Guo, X., Li, Z., Li, B., Li, Z., Li, R., et al. (2015). A systematic investigation based on microRNA-mediated gene regulatory network reveals that dysregulation of microRNA-19a/Cyclin D1 axis confers an oncogenic potential and a worse prognosis in human hepatocellular carcinoma. RNA Biol. 12, 643-657. doi: 10.1080/15476286.2015.1022702

Zhang, Y., Wei, W., Cheng, N., Wang, K., Li, B., Jiang, X., et al. (2012). Hepatitis C virus-induced up-regulation of microRNA-155 promotes hepatocarcinogenesis by activating Wnt signaling. Hepatology 56, 1631-1640. doi: 10.1002/hep.25849

Zhang, Y., Zheng, D., Xiong, Y., Xue, C., Chen, G., Yan, B., et al. (2014). miR-202 suppresses cell proliferation in human hepatocellular carcinoma by downregulating LRP6 post-transcriptionally. FEBS Lett. 588, 1913-1920. doi: 10.1016/j.febslet.2014.03.030

Zhao, J. J., Lin, J., Zhu, D., Wang, X., Brooks, D., Chen, M., et al. (2014). miR30-5p functions as a tumor suppressor and novel therapeutic tool by targeting the oncogenic Wnt/beta-catenin/BCL9 pathway. Cancer Res. 74, 1801-1813. doi: 10.1158/0008-5472.CAN-13-3311-T

Zheng, F., Liao, Y. J., Cai, M. Y., Liu, T. H., Chen, S. P., Wu, P. H., et al. (2015). Systemic delivery of microRNA-101 potently inhibits hepatocellular carcinoma in vivo by repressing multiple targets. PLoS Genet. 11:e1004873. doi: 10.1371/ journal.pgen.1004873

Conflict of Interest Statement: The authors declare that the research was conducted in the absence of any commercial or financial relationships that could be construed as a potential conflict of interest.

Copyright (C) 2018 Nie, Liu, Chen and Wang. This is an open-access article distributed under the terms of the Creative Commons Attribution License (CC BY). The use, distribution or reproduction in other forums is permitted, provided the original author(s) and the copyright owner are credited and that the original publication in this journal is cited, in accordance with accepted academic practice. No use, distribution or reproduction is permitted which does not comply with these terms. 